\title{
Earmold Foreign Bodies in the Middle Ear Necessitating Surgical Removal: Why Otology Specialists Should Screen Candidates for Hearing Aids
}

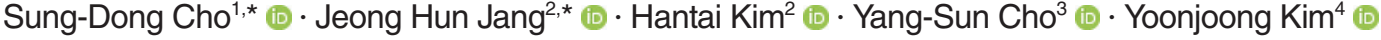 \\ Ja-Won Koo ${ }^{1}$ (iD $\cdot$ Jae-Jin Song ${ }^{1}$ (i) \\ ${ }^{1}$ Department of Otorhinolaryngology-Head and Neck Surgery, Seoul National University Bundang Hospital, Seongnam; \\ ${ }^{2}$ Department of Otolaryngology, Ajou University School of Medicine, Suwon; ${ }^{3}$ Department of Otorhinolaryngology-Head and Neck Surgery, \\ Samsung Medical Center, Sungkyunkwan University School of Medicine, Seoul; ${ }^{4}$ Department of Otolaryngology-Head and Neck Surgery,
} Chungnam National University College of Medicine, Daejeon, Korea

The fitting of hearing aids (HAs) usually requires the creation of an earmold that assists device fixation in the ear canal and reduces feedback. Mold construction is preceded by acquiring an impression of the external auditory canal. Although impression molding is generally safe, it may trigger an inflammatory reaction and deterioration of aeration if the impression mixture accidentally becomes lodged in the middle ear. Herein, we present a case series of four patients with mold foreign bodies of the middle ear that required surgical removal, with a literature review. We retrospectively analyzed four patients who presented with severe complications caused by inappropriate earmold fitting who required immediate surgical interventions at three different centers (cases 1 and 2 at Seoul National University Bundang Hospital [Fig. 1], case 3 at Ajou University Hospital [Fig. 2A-F], and case 4 at Samsung Medical Center [Fig. 2G-L]) from October 2012 to April 2019. The Institutional Review Board of Clinical Research Institute of Seoul National University Bundang Hospital approved this retrospective study (IRB No. B-2004-608-111) and waived the requirement for informed

\footnotetext{
- Received May 9, 2020

Revised July 29, 2020

Accepted August 25, 2020

- Corresponding author: Jae-Jin Song

Department of Otorhinolaryngology-Head and Neck Surgery, Seoul

National University Bundang Hospital, 82 Gumi-ro 173beon-gil,

Bundang-gu, Seongnam 13620, Korea

Tel: +82-31-787-7408, Fax: +82-31-787-4057

E-mail: jjsong96@snubh.org

*These authors contributed equally to this work.
}

consent.

From patients' medical records, we collected information on preoperative status, including otoendoscopic findings, audiometry results, and temporal bone computed tomography (TBCT) findings. Postoperative follow-up otoendoscopic and audiometric results were analyzed using a previously described method [1-5].The subjects' information is summarized in Supplementary Table 1, Figs. 1 and 2. All patients had histories of prior chronic otitis media, but had not been seen by otology specialists prior to HA-mold preparation. On physical examination, all four patients showed tympanic membrane perforations with visible middle ear foreign bodies. The TBCT findings of all subjects showed silicone filling the middle ears and extending to the Eustachian tubes. In cases 1 (Fig. 1A-F) and 3 (Fig. 2A-F), the mold material filled the intercrural areas of the stapes. Case 2 (Fig. 1G-L) exhibited extension of the mold material to the mastoid antrum.

Three patients underwent intact canal wall mastoidectomy to remove the molds, whereas the other one (case 1) underwent transcanal foreign body removal, tympanoplasty, and piston wire prosthesis insertion after stapedectomy via the retroauricular approach due to subluxation of the stapes footplate with partial fracture of the anterior half of the footplate developed despite rapid and meticulous dissection of the material. Postoperatively, case 1 suffered from positional vertigo, which improved over 4 months postoperatively. Case 2 developed ipsilesional House-Brackmann grade II facial nerve palsy (FNP) when an attempt was made to remove the mold material at a local clinic; the FNP persisted even after total foreign body removal. On

Copyright @ 2021 by Korean Society of Otorhinolaryngology-Head and Neck Surgery.

This is an open-access article distributed under the terms of the Creative Commons Attribution Non-Commercial License (https://creativecommons.org/licenses/by-nc/4.0) which permits unrestricted non-commercial use, distribution, and reproduction in any medium, provided the original work is properly cited. 

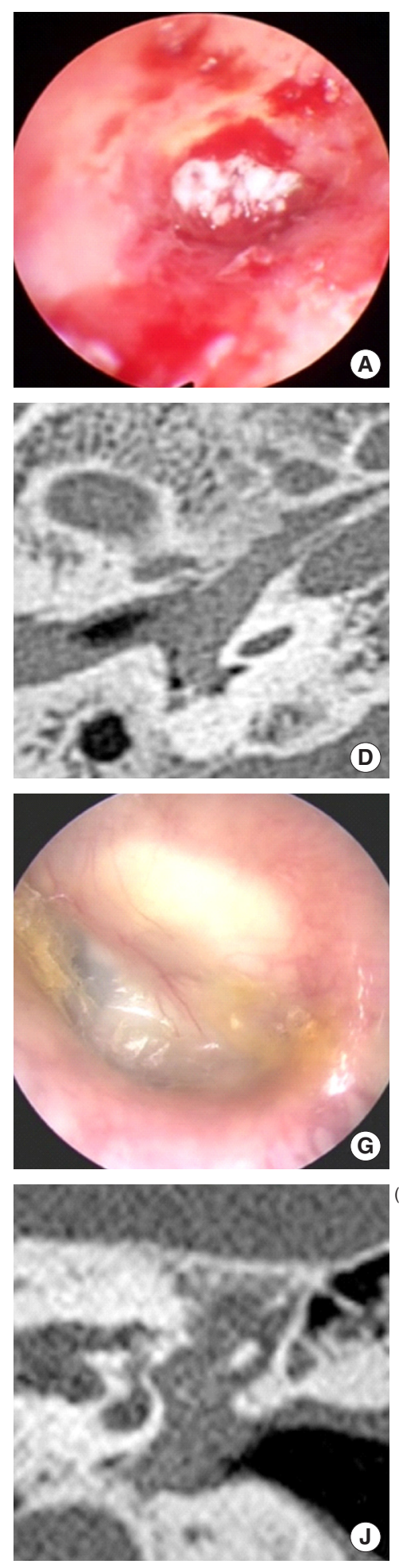
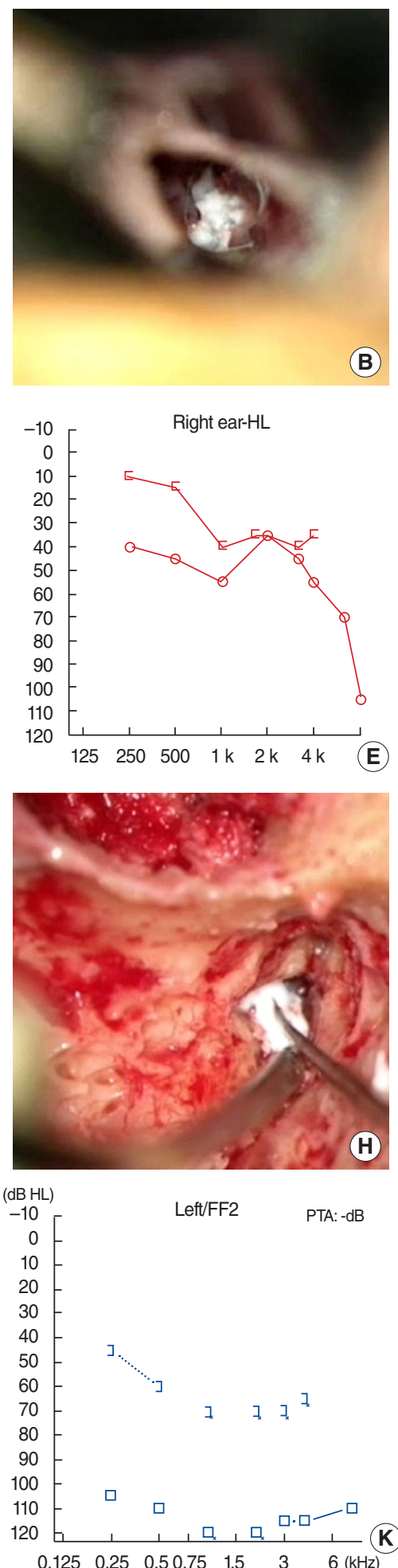
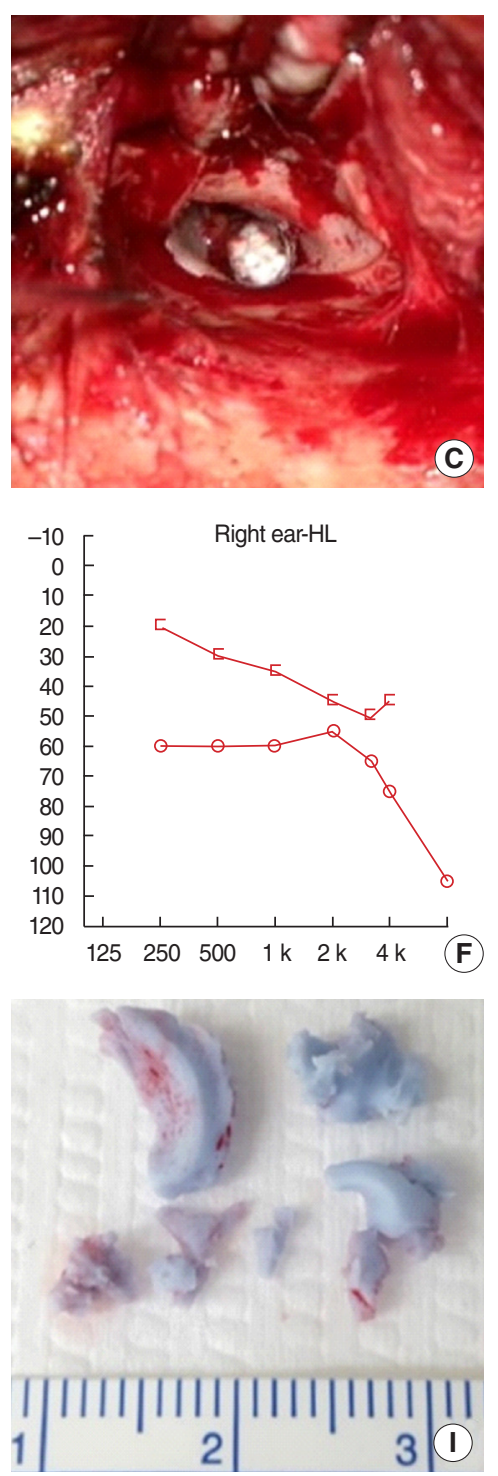

Left ear-HL

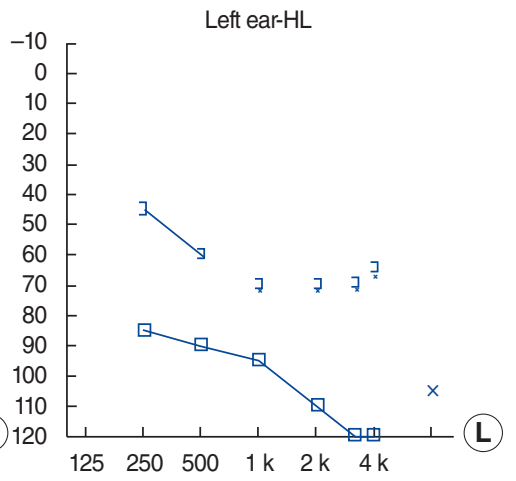

Fig. 1. Summary of cases 1 and 2. (A-F) Case 1. (A) An endoscopic view revealed earmold impression material filling the middle ear. (B) After transcanal visualization of the middle ear, $(C)$ the foreign body was removed via the retroauricular approach. (D) The silicone material extended to the Eustachian tube and the round window niche. Preoperative (E) and postoperative 3-month (F) pure-tone audiometry data. (G-L) Case 2. (G) An endoscopic examination revealed a pseudomembrane external to the earmold impression material located in the middle ear. $(\mathrm{H})$ The foreign body in the middle ear and mastoid antrum was removed via a combined transcanal-transmastoid approach. (I) The silicone material was totally removed. $(\mathrm{J})$ The silicone material extended to the Eustachian tube on a coronal computed tomography scan. Preoperative (K) and postoperative 5-month (L) pure-tone audiometry (PTA) data. 

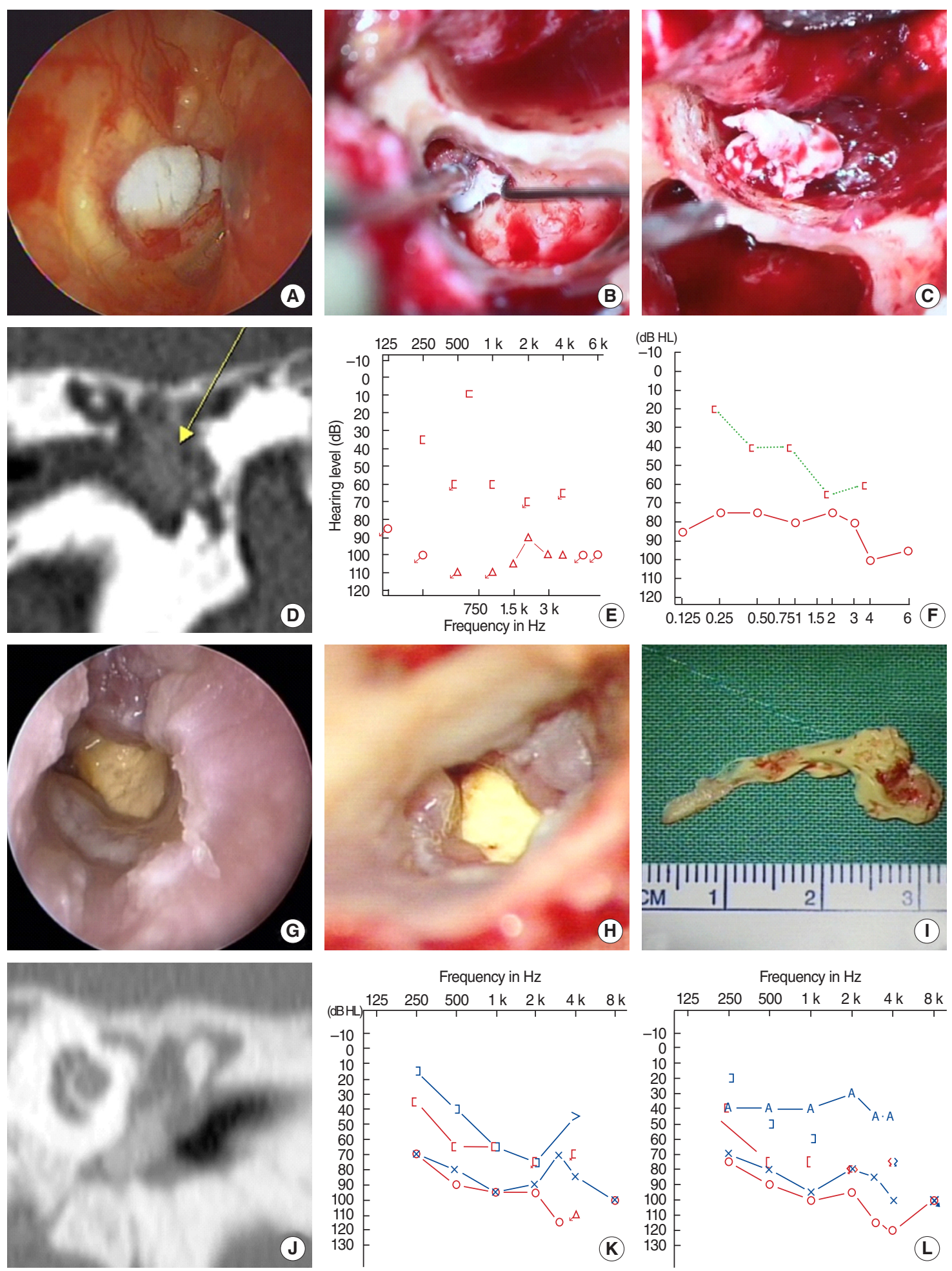

Fig. 2. Summary of cases 3 and 4. (A-F) Case 3. (A) Earmold impression material filling the middle ear can be seen through the perforated tympanic membrane. $(B, C)$ The foreign body was removed en bloc via a combined transcanal-transmastoid approach. (D) Silicone material (yellow arrow) in the middle ear. Preoperative (E) and postoperative 3-month (F) pure-tone audiometry data. (G-L) Case 4. (G) Earmold impression material filling the middle ear can be seen through the perforated tympanic membrane. $(\mathrm{H}, \mathrm{I})$ The foreign body was removed en bloc via a combined transcanal-transmastoid approach. $(\mathrm{J})$ The silicone material in the middle ear extended to the Eustachian tube on a coronal computed tomography scan. Preoperative $(\mathrm{K})$ and postoperative 2-year $(\mathrm{L})$ pure-tone audiometry data. 
pure tone audiometry performed 1 year postoperatively, case 2 exhibited ipsilesional profound $(>90 \mathrm{~dB})$, sensorineural hearing loss and the other three cases showed mixed hearing loss with thresholds ranging from 60 to $85 \mathrm{~dB}$.

In the current case series, we reviewed four patients with histories of tympanic membrane perforation who presented with middle ear foreign bodies composed of silicone mold material that required surgical removal. The earmold materials were found not only in the middle ear, but also in the sinus tympani, mastoid antrum, and Eustachian tube. Even after successful removal of the material, all patients exhibited profound or severe mixed hearing loss. A total of 36 cases were analyzed in a recent case series that included a literature review [6]. Of the 36 patients, 33 underwent surgical removal of the impression material. Previous studies have reported postoperative complications such as labyrinthitis and lateral semicircular canal dehiscence or persistent hearing loss. Although all four of our cases were managed by three experienced otological surgeons, delayed referral to tertiary care centers and extensive invasion into or around critical structures precluded successful audiological outcomes even after rapid surgical interventions. Furthermore, the FNP in case 2 and other cases of vestibular complications lacking any round or oval window damage described in the literature suggest that the material may be toxic to the cochleovestibular system after contact with the labyrinth. In this regard, early referral to otology specialists and prompt surgical intervention to remove the entire foreign body without damaging critical middle ear structures is of the utmost importance if it is possible that impression material has entered the middle ear.

According to U.S. regulations, HA dispensers should consult otologists before fitting HAs in patients exhibiting external ear deformities, otorrhea, sudden hearing loss, dizziness, an air-bone gap $>15 \mathrm{~dB}$, cerumen impaction, or otalgia [7]. However, even in the United States, HA mold impression material-related complications have been regularly reported [7], possibly because of non-adherence to federal and state laws. Notably, the Over-theCounter Hearing Aid Act 2016, which removed the need for medical evaluation, has exacerbated the problem. In Japan, a certification program for HA dispensers was developed, but the system is not mandatory and HA impression-related complications continue to be reported [8]. HA molding material-related complications have previously been reported in South Korea $[9,10]$, which lacks any specific training program for HA-dispensing audiologists in terms of otological examinations or indications for the referral of HA candidates with external or middle ear pathologies.

HAs are prescribed either after careful examination by otolaryngologists or directly by audiologists in South Korea. Molding materials may trigger serious complications that worsen residual hearing and preclude HA-assisted auditory rehabilitation, as in our current cases. HA-dispensing audiologists must always send patients to otologists if there is any history or current suspicion of ear infection. Although all four patients had histories of chronic otitis media, none was seen by an otologist. Additionally, all four subjects were referred to tertiary care centers at least 2 weeks after the development of molding material-related complications. Therefore, a non-punitive reporting system expediting urgent surgical management is essential.

Our current case series shows that significant complications may develop when taking earmold impressions. Legally mandated referral of HA candidates with possible external or middle ear problems to otology specialists, and non-punitive reporting of subjects with impression material-related complications, are essential to prevent irreversible complications that may develop when fitting HAs.

\section{CONFLICT OF INTEREST}

No potential conflict of interest relevant to this article was reported.

\section{ACKNOWLEDGMENTS}

This work was supported by a National Research Foundation of Korea (NRF) grant funded by the Korean government (MSIT) (grant no. NRF-2019R1A2C2004941).

\section{ORCID}

Sung-Dong Cho https://orcid.org/0000-0002-7140-6863

Jeong Hun Jang https://orcid.org/0000-0003-1707-5714

Hantai Kim https://orcid.org/0000-0002-5020-7814

Yang-Sun Cho https://orcid.org/0000-0001-9180-2903

Yoonjoong Kim https://orcid.org/0000-0002-9589-9244

Ja-Won Koo https://orcid.org/0000-0002-5538-2785

Jae-Jin Song https://orcid.org/0000-0002-6631-3232

\section{AUTHOR CONTRIBUTIONS}

Conceptualization: JJS. Data curation: SDC, HK. Formal analysis: SDC, JHJ, YSC, YK, JWK. Funding acquisition: JJS. Methodology: JHJ. Project administration \& Visualization: SDC. Writingoriginal draft: SDC, JJS. Writing-review \& editing: SDC, JJS.

\section{SUPPLEMENTARY MATERIALS}

Supplementary materials can be found via https://doi.org/10. 21053/ceo.2020.00850. 


\section{REFERENCES}

1. Shim YJ, Bae YJ, An GS, Lee K, Kim Y, Lee SY, et al. Involvement of the internal auditory canal in subjects with cochlear otosclerosis: a less acknowledged third window that affects surgical outcome. Otol Neurotol. 2019 Mar;40(3):e186-90.

2. Lee SY, Song SK, Park SJ, Park HG, Choi BY, Koo JW, et al. Jugular bulb resurfacing with bone cement for patients with high dehiscent jugular bulb and ipsilateral pulsatile tinnitus. Otol Neurotol. 2019 Feb;40(2):192-9.

3. Han SA, Choe G, Kim Y, Koo JW, Choi BY, Song JJ. Beware of a fragile footplate: lessons from ossiculoplasty in patients with ossicular anomalies related to second pharyngeal arch defects. J Clin Med. 2019 Dec;8(12):2130.

4. Han JJ, Ridder D, Vanneste S, Chen YC, Koo JW, Song JJ. Pre-treatment ongoing cortical oscillatory activity predicts improvement of tinnitus after partial peripheral reafferentation with hearing aids.
Front Neurosci. 2020 May;14:410.

5. Huh G, BaeYJ, Woo HJ, Park JH, Koo JW, Song JJ.Vestibulocochlear symptoms caused by vertebrobasilar dolichoectasia. Clin Exp Otorhinolaryngol. 2020 May;13(2):123-32.

6. Verdam F, Tange R, Thomeer H. Impression material in the external and middle ear: an overview of the literature and a stepwise approach for removal. J Int Adv Otol. 2016 Dec;12(3):345-52.

7. Meyers JA, Ardeshirpour F, Hilton CW, Levine SC. Complication from hearing aid mold material: a case report and review of legal matters. Am J Otolaryngol. 2013 Nov-Dec;34(6):739-42.

8. SugiuchiT, Kodera K, Zusho H,AsanoY, Kanesada K, Hayashida M, et al. Complications resulting from taking ear impressions. Nihon Jibiinkoka Gakkai Kaiho. 2015 Aug;118(8):1058-67.

9. Lee HM, Yi KI, Jung JH, Lee IW. Hearing aid silicone impression material as a foreign body in the middle ear. Am J Otolaryngol. 2017 Jan-Feb;38(1):108-11.

10. Lee DH, Cho HH. Otologic complications caused by hearing aid mold impression material. Auris Nasus Larynx. 2012 Aug;39(4):411-4. 